Trapeziectomy is a surgical treatment for patients with intractable base of thumb $O A$ and is a good source of hand OA cartilage.

Objectives: The aim of this study was to investigate the expression of atRAdependent and inflammatory genes in the cartilage of patients and to examine these with respect to the presence/absence of polymorphic ALDH1A2 variants. We also investigate the regulation of atRA upon cartilage injury and its effect on injury-induced inflammatory gene regulation.

Methods: We collected 26 trapeziectomy samples, and dissected the cartilage within one hour of collection. Genomic DNA was extracted for the identification of the two common variants (SNP rs4238326 and SNP rs3204689). Expression levels of atRA-dependent and inflammatory genes were tested by RT-PCR.

Healthy cartilage was obtained from femoral heads of 5-week-old mice and porcine metacarpophalangeal joints of 3-6 months old pigs. Gene regulation upon cartilage injury was tested. To examine the role of specific injury-induced pathways, porcine joints were pre-injected with selective inhibitors to: TGF $\beta$, FGF2R, TAK1 and CYP26, 1 hour prior to injury.

Results: Polymorphic variants in ALDH1A2 were common in this patient population and we identified 8 patients homozygous for both variants, and 5 patients who were wild type for both variants. mRNA levels of ALDH1A2 and atRA-dependent gene CYP19A were significantly lower in the homozygous group compared to wild type (figure 1). There were also trends in the regulation of several other atRAdependent genes. Conversely, inflammatory genes such as HAS1, TSG6 and ADAMTS5 showed a general increase in homozygous patients.

Cartilage injury in both porcine and murine tissue led to a rapid down-regulation of atRA-dependent genes (CYP26s and RARs). Injecting joints with a potent TAK1 inhibitor prevented the drop of atRA-dependent genes. Prior injection of the joint with a CYP26 (enzymes that normally break down atRA) inhibitor, restored levels of atRA-dependent genes after injury and suppressed injury-induced inflammation genes.

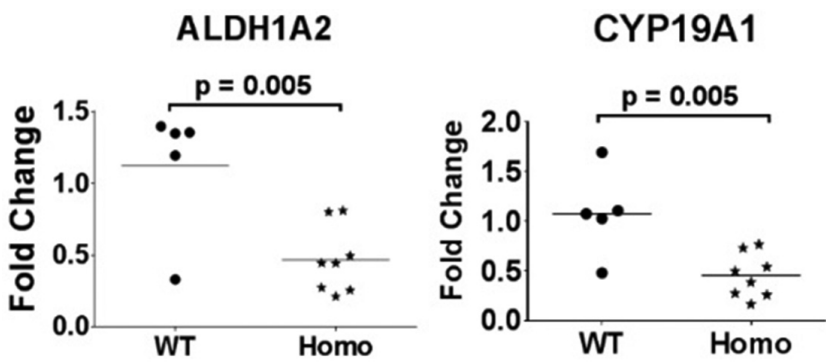

Conclusions: Polymorphic variants in ALDH1A2 are associated with significantly lower levels of ALDH1A2 and CYP19A1 mRNA in hand OA cartilage. Adult articular cartilage constitutively produces atRA, and this is strongly suppressed by mechanical injury through TAK1 activation. Preventing the drop in cellular atRA upon injury, by pre-incubating the joint with a CYP26 inhibitor, restores atRA levels and leads to a reduction in inflammatory gene regulation. These results indicate that atRA plays an important anti-inflammatory role in cartilage and provides a potential novel therapeutic strategy to treat hand $O A$.

Disclosure of Interest: None declared

DOI: 10.1136/annrheumdis-2018-eular.3647

\section{OP0262 1 DISEASE MODIFYING EFFECTS OF THE CANINE IL4-10 FUSION PROTEIN IN THE CANINE GROOVE MODEL OF OSTEOARTHRITIS}

E.M. Van Helvoort ${ }^{1}$, J. Popov-Celeketic ${ }^{1}$, K. Coeleveld ${ }^{1}$, A. Doornenbal ${ }^{2}$, M. Tryfonidou ${ }^{2}$, C. Wijne ${ }^{1}$, F. Lafeber ${ }^{1}$, S. Mastbergen ${ }^{1} .{ }^{1}$ Rheumatology and Clinical Immunology, University Medical Centre Utrecht, Utrecht University; ${ }^{2}$ Department of Clinical Sciences of Companion Animals, Faculty of Veterinary Medicine, Utrecht University, Utrecht, Netherlands

Background: An ideal disease modifying osteoarthritis (OA) drug should have analgesic, chondroprotective and anti-inflammatory effects. A fusion protein of Interleukin 4 and 10 (IL4-10FP) might have these effects.

Objectives: This study evaluates the effects of canine IL4-10FP (cIL4-10FP) in the canine Groove model of OA.

Methods: In 8 skeletally mature dogs, knee OA in the right leg was induced according to the Groove model. After 6 weeks of OA development, intra-articular injections in the affected knee with either PBS (500 $\mu$; n=4) or clL4-10FP (10 $\mu \mathrm{g} /$ $500 \mu \mathrm{l} ; \mathrm{n}=4)$ were given weekly for 10 weeks. The contra-lateral joints served as a healthy control.

Force plate analysis (FPA) was used to determine joint loading as a surrogate marker of pain. The ratio of the affected over the contra-lateral control joint was calculated for each dog for each time point. FPA was performed before OA induction to determine baseline values, and before and 24 hours after the 1 st, 6 th and 9th intra-articular injection. A linear mixed model was used to evaluate effects of injections. After 10 weeks of treatment dogs were euthanized and tissue samples were harvested. Serum Immunoglobulin G (IgGs) titers against clL4-10FP were evaluated to check for potential antibody formation.

Cartilage proteoglycan content and release of proteoglycans were determined ex vivo by Alcian Blue assay. Synovial inflammation was evaluated by HE-staining using OARSI grading. Changes in outcomes in the affected/treated joints compared to contra-lateral control joints were calculated.

Results: After OA induction a clear reduction in joint loading (increase in pain) was found (standing force and braking force). After clL4-10FP injections these forces increased toward normalisation (clL4-10FP vs PBS group, $p=0.002$ and $p=0.01$, respectively). No IgG elevation was detected after 10 injections. Compared to contra-lateral controls, proteoglycan content of OA PBS injected knees suggested tissue degeneration (27 vs $34 \mathrm{mg} / \mathrm{g}$ ). In the clL4-10FP treated group proteoglycan content in right knees was not different from the contralateral controls ( 33 vs $31 \mathrm{mg} / \mathrm{g}$ ). The mean change in proteoglycan content compared to contra-lateral controls was different with a $p$ value of 0.057 (figure 1). A similar pattern was found for the change in release of proteoglycans from the cartilage, which was less increased in the cIL4-10FP group compared to the PBS group $(0.4 \%$ vs $3.0 \% ; p=0.029$, figure 1 ).

Synovial inflammation was mild (characteristic of this model) and did not change after intra-articular injections $(0.4$ and -1.3 points out of 18 for clL4-10FP and PBS respectively).
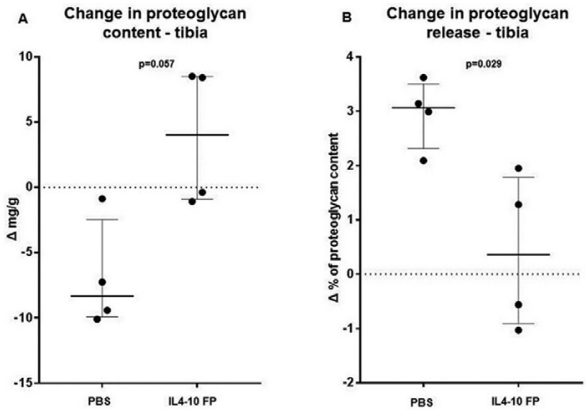

Abstract OP0262 - Figure 1 Chondroprotective effects of clL4-10 fusion protein. (A) change in proteoglycan content between left (control) and right (injected) knees. Values are expressed per animal, representing a mean of 8 samples, and as median $\pm I Q R$. (B) change in release of proteoglycans between left (control) and right (injected) knees. Values are expressed per animal, representing a mean of 8 samples, and as median \pm IQR.

Conclusions: Repetitive intra-articular injection with canine IL4-10FP in dogs did not lead to antibody formation. Dogs treated with clL4-10FP showed improved joint loading compared to PBS treated dogs, reflecting an analgesic effect. Recovery of proteoglycan content and normalisation of release in clL4-10FP treated dogs indicate a chondroprotective effect. Synovial inflammation in the Groove model was too mild to be changed significantly. These results clearly warrant further research to develop IL4-10FP as a DMOAD.

Acknowledgements: This work is supported by the Dutch Arthritis Foundation project NR12-2-202

Disclosure of Interest: None declared

DOI: 10.1136/annrheumdis-2018-eular.1665

\section{OP0263 CLAUDIN-11 REGULATES BONE HOMEOSTASIS VIA BIDIRECTIONAL EPHB4-EPHRINB2 SIGNALLING}

C.-H. Lee ${ }^{1^{*}}$, J.M. Baek ${ }^{2}$, Y.J. Choi ${ }^{3}$, W.-H. Yoo ${ }^{3}$, M.S. Lee ${ }^{4}$, J.-Y. Kim² ${ }^{1}$ Wonkwang University Hospital; ${ }^{2}$ Anatomy, Wonkwang University, Iksan; ${ }^{3}$ Internal Medicine, Chonbuk National University Hospital, Jeonju; ${ }^{4}$ Internal Medicine, Wonkwang University Hospital, Iksan, Korea, Republic Of

Background: Claudins (Cldns) are well-established components of tight junc tions (TJs) that play a pivotal role in the modulation of paracellular permeability Several studies have explored the physiologic aspects of Cldn family members in bone metabolism. However, the effect of Cldn11, a major component of centra nervous system myelin, on bone homeostasis has not been reported.

Objectives: This study was performed to identify the effects of Cldn on bone metabolism via regulation of osteoclast and osteoblast differentiation and their function

Methods: We performed various in vitro and in vivo studies using gain- and loss of function of Cldn11 that is belong to the Cldn group. Osteoclast formation from bone marrow cells (BMC) and Osteoblast formation was evaluated in specific condition with over-expression or down- regulation of Cldn11. The expression of osteoclast associated gene and osteoblast related gene mRNA were assessed 